\title{
Osteogenic differentiation of human mesenchymal stem cells in the absence of osteogenic supplements: a surface-roughness gradient study
}

Ana B. Faia-Torres 1,2,3, Mirren Charnley3,4,5, Tolga Goren³, Stefanie Guimond-Lischer6, Markus Rottmar ${ }^{6, a}$, Katharina Maniura-Weber ${ }^{6, b}$, Nicholas D. Spencer ${ }^{3}$, Rui L. Reis ${ }^{1,2}$, Marcus Textor ${ }^{3}$, Nuno M. Neves ${ }^{*}, 1,2$

13B's Research Group - Biomaterials, Biodegradables and Biomimetics, University of Minho, Avepark - Parque de Ciência e Tecnologia, Zona Industrial da Gandra, 4805017 Barco GMR, Portugal 2ICVS/3B's - PT Government Associate Laboratory, Guimarães, Portugal Laboratory for Surface Science and Technology, Department of Materials, ETH Zurich, $\mathrm{CH}-8093$ Zürich, Switzerland

${ }^{3}$ Laboratory for Surface Science and Technology, Department of Materials, ETH Zurich, $\mathrm{CH}-8093$ Zürich, Switzerland

${ }^{4}$ Centre for Micro-Photonics, and ${ }^{5}$ ndustrial Research Institute Swinburne, Faculty of Science, Engineering and Technology, Swinburne University of Technology, Victoria 3122, Australia.

${ }^{6}$ Laboratory for Materials-Biology Interactions, Empa Swiss Federal Laboratories for Materials Science and Technology, CH-9014 St. Gallen, Switzerland

*E-mail: nuno@dep.uminho.pt; Tel.: +351 253510 905; fax: +351 253510909. 


\section{Abstract}

The use of biomaterials to direct osteogenic differentiation of human mesenchymal stem cells (hMSCs) in the absence of osteogenic supplements is thought to be part of the next generation of orthopedic implants. We previously engineered surface-roughness gradients of average roughness $\left(R_{a}\right)$ varying from the sub-micron to the micrometer range $(\sim 0.5-4.7 \mu \mathrm{m})$, and mean distance between peaks $\left(R S_{m}\right)$ gradually varying from $\sim 214 \mu \mathrm{m}$ to $33 \mu \mathrm{m}$. Here we have screened the ability of such surface-gradients of polycaprolactone to influence the expression of alkaline phosphatase (ALP), collagen type 1 (COL1) and mineralization by hMSCs cultured in dexamethasone (Dex)-deprived osteogenic induction medium (OIM) and in basal growth medium (BGM). $\mathrm{Ra}_{a} \sim 1.53$ $\mu \mathrm{m} / \mathrm{RS}_{\mathrm{m}} \sim 79 \mu \mathrm{m}$ in Dex-deprived OI medium, and $\mathrm{R}_{\mathrm{a}} \sim 0.93 \mu \mathrm{m} / \mathrm{RS}_{\mathrm{m}} \sim 135 \mu \mathrm{m}$ in BGM consistently showed higher effectiveness at supporting the expression of the osteogenic markers ALP, COL1 and mineralization, compared to the tissue culture polystyrene (TCP) control in complete OIM. The superior effectiveness of specific surfaceroughness revealed that this strategy may be used as a compelling alternative to soluble osteogenic inducers in orthopedic applications featuring the clinically relevant biodegradable polymer polycaprolactone.

\section{Keywords}

Biomaterials, high-throughput screening (gradient), microstructures, mesenchymal stem cells, osteogenesis

\section{Introduction}

The bone tissue engineering field investigates viable options to replace permanent medical implants, which are commonly applied to fill bone defects. The ideal substitute 
aims at inducing new functional tissue and at the material resorption after normal tissue function has been restored $[1,2]$. The resorbable characteristic of the implant-material is particularly important in pediatric trauma, given that the skeleton of the pediatric patients is not fully developed and the implant size will eventually mismatch the growing body, which would require secondary corrective surgeries. Polycaprolactone $(P C L)$ is a resorbable polymer, which has been thoroughly studied for its biocompatibility, degradation behavior, mechanical properties and easy manipulation into different scaffold-architectures by a wide range of processing techniques [ 3-7]. FDA-approved for its use in several medical devices, PCL is a particularly interesting option to explore for low-load-bearing applications, such as craniofacial bone solutions $[4,8]$.

The cell seeding of the scaffolding-material with mesenchymal stem cells (MSCs) has been reported to potentiate the scaffold's positive effect $[9,10]$. Quite frequently, however, synthetic polymers, such as PCL, do not demonstrate particular bioactivity/affinity [11], which hinders the interaction of cells and tissues with the implant, ultimately limiting the effectiveness of the implant-integration in the body [12]. In order to obtain a specific cell response, tailoring of physical or chemical surface properties of the material is a valuable tool [12-15]. Among the different surface modifications that can be used to improve the materials' bioactivity, altering the surface roughness has been regarded as one of the most relevant approaches promoting osteointegration [16]. This may be due to the long-term interlock provided by rough surfaces at the cell-material interface, while surface chemistry seems to more strongly influence the short-term cell adhesion [17-19]. 
Human bone marrow MSCs (hBM-MSCs) are an accessible adult cell population [20], which can be conveniently expanded in vitro [21], having the ability to give rise to multiple stromal lineages, such as the osteoblastic (bone), adipogenic (fat) and chondrogenic (cartilage) [22]. The osteogenic induction of MSCs in vitro may not only guarantee the desired lineage-commitment prior to implantation but also reduce the interval between the implantation and subsequent in situ osteogenesis [23]. Furthermore, culturing the cells in vitro contributes for the maturation of the interactions between cells and scaffold, which has been correlated with an improved the efficacy of the cell-scaffold hybrid construct [24]. It is widely accepted that soluble factors, such as those present in the cell culture media, considerably influence MSC self-renewal, proliferation and differentiation [25]. Dexamethasone (Dex) is a potent glucocorticoid commonly used in the osteogenic induction medium (OIM) of human MSCs [25], and has also been included in drug delivery scaffolds to support osteogenesis [26]. However, as it has contradictory behavioral effects at different concentrations, treatment length and timing of supplementation, with negative consequences for osteogenesis, the optimal use of this agent in the osteogenic process is still unclear $[27,28]$. Furthermore, Dex has a negative influence in the recruitment of resident MSCs to the implant site in vivo [29], hindering the crosstalk between the resident and the exogenous cell population, which is known to significantly contribute to the success of the tissue-engineered approach [30]. Therefore, an alternative to Dex could be of particular interest for the biomedical community.

The ideal scaffold for bone-related approaches should be able to trigger and to continuously support the human MSC commitment towards the desired lineage, 
avoiding the need to expose the cells to osteogenic-inducer bioagents, which inherently have short-term availability and complex interactions. In such a task, surface roughness influencing the osteogenic differentiation of hBM-MSCs could offer a significant, finely tailored, easy-to-manufacture and inexpensive option. Despite the scientific interest and relevance of this topic, few reports address the osteogenic potential of physical (or chemical) cues in the partial or total absence of standard soluble differentiation supplements in the medium $[31,32]$. For instance, the effect of surface-roughness in biodegradable polymer substrates, in absence of osteogenic-inducer bioagents, has not yet been examined. We previously showed that varying the surface-roughness strongly influences the osteogenic commitment potential of hBM-MSCs cultured on PCL, in the presence of the standard OIM [33]. Given the remarkable results obtained in comparison to the reference material, tissue culture polystyrene (TCP), we now hypothesized that the osteogenic differentiation of hBM-MSCs could be accomplished by specific surface-roughness in osteogenic conditioned medium deprived of Dex, and even in total absence of osteogenic inducting supplementation (i.e., in basal growth medium). To test this hypothesis we cultured hBM-MSCs on similar surface-roughness gradients, varying from the micro- to sub-micrometer roughness average range. Early and late markers of osteogenesis, such as alkaline phosphatase (ALP), collagen type 1 (COL1) and mineralization were assessed. The results where then compared to TCP in standard OIM (TCP+), Dex-deprived OIM (TCP-) and basal growth medium (TCP-B). We found that, in comparison to the positive control, specific surface-roughness parameters $\left(R_{a} / R_{m}\right)$ have the ability to trigger and support significantly greater mineralization of the deposited extracellular matrix, even in basal growth medium. Thus, 
the data validates the effectiveness of the appropriate surface roughness range of PCL in the osteogenic modulation of hBM-MSCs. To the best of our knowledge this is the first systematic study of the influence of micro- to sub-micrometer roughness values of a clinically relevant biodegradable polymer on the osteogenic fate of stem cells, in both partial and total absence of standard osteogenic inducing supplements in vitro.

\section{Materials and Methods}

\subsection{Materials}

Surface-roughness gradients on polycaprolactone (PCL) were produced and characterized as previously detailed [33]. In brief, polyvinylsiloxane (PVS) was used to obtain (negative) replicas of the original gradient created by sandblasting and chemical polishing. Then, the PVS masters were used to produce, by hot-embossing, the (positive) gradient replica in solvent-cast PCL membranes. The PCL surface-roughness gradients were sterilized by air-plasma at 0.1 mbar for 34 s (PDC-32G Harrick Scientific). The PCL roughness gradients were then analyzed by optical profilometry (Plu NEOX, Sensofar, Spain) and scanning electron microscopy (SEM; LEO 1350). The quantitative amplitude and spacing parameters $\left(R_{a}\right.$ and $\left.R S_{m}\right)$ of the gradient features

were calculated following the ISO 4287 standard. The sharpness (kurtosis, Sku) and the degree of symmetry of the surface in terms of dominance of peaks and valleys (skewness, Ssk) were determined in accordance with the ISO 25178 standard.

\subsection{Isolation and Expansion of hBM-MSCs}

Femur-derived bone marrow was used to isolate the mesenchymal stem cells used in this work, as previously described [51]. The human samples were collected, following 
informed written consent, from three independent donors (78-82 years old) with the approval from the ethics committee of the Kantonspital St. Gallen, Switzerland (EKSG

08/14). During cell expansion the basal growth medium (alpha ( $\alpha$ )-MEM supplemented with 10\% Fetal Bovine Serum (FBS; Lonza), 1\% penicillin-streptomycine-neomycin antibiotic mixture (100 x) (PSN; Gibco) and $1 \mathrm{ng} \mathrm{mL}^{-1}$ fibroblast growth factor 2 (FGF2; Sigma, F0291)) was changed twice a week. The cells were sub-cultured prior to confluence, and were used in the experiments at cell passages one or two.

\subsection{Seeding and Osteogenic Differentiation of hBM-MSCs}

The PCL surface-roughness gradients and tissue culture polystyrene (TCP) samples were seeded with 3000 cells $\mathrm{cm}^{-2}$ in basal growth medium. The cells were kept at 37 ${ }^{\circ} \mathrm{C} / 5 \% \mathrm{CO}_{2}$. For the study of the osteoblastic differentiation of the hBM-MSCs, $24 \mathrm{~h}$ later the medium was replaced in the samples according to the culture conditions set as follows: 1) TCP in complete osteogenic induction medium (OIM) ( $\alpha$-MEM supplemented with 10\% FBS, 1\% PSN, 10 nM dexamethasone (Sigma, D4902), 50 mM ascorbate-2phosphate (Sigma, A8960), 2mM beta-glycerophosphate (Sigma,G9891) and $10 \mathrm{nM}$ 1,25-dihydroxyvitamine D3 (Sigma, D1530)), 2) TCP in dexamethasone-deprived OIM, 3) TCP in basal growth medium (as above detailed), 4) surface-roughness gradients in dexamethasone-deprived OIM, and 3) surface-roughness gradients in basal growth medium. The medium was replaced every 2 to 3 days, for the 21 -day study. Three replicates per condition and time point were used, and three independent studies were performed using single-donor cell populations per study. 


\subsection{Immunodetection of osteogenic markers in the cultured cell population}

Six time points were chosen to study the osteogenic commitment of hBM-MSCs: days $1,4,7,10,14$ and 21 . The cells were fixed and permeabilized with a $4 \%$ paraformaldehyde/0.2\% Triton X-100 solution, for $8 \mathrm{~min}$ at room temperature (RT). Following fixation, the samples were maintained in PBS at $4^{\circ} \mathrm{C}$, until the simultaneous labeling of all samples was possible. Incubation of primary antibodies against alkaline phosphatase (ALP; 1:1000, Developmental Hybridoma Bank, B4-78) and collagen type 1 (COL1; 1:1000, Sigma, St Louis, MO, USA, C 2456) was performed for $1 \mathrm{~h}$ at RT, upon incubation of the samples with $1 \%$ fetal calf serum $/ 5 \%$ goat serum, for $30 \mathrm{~min}$ at RT, to prevent non-specific binding. Secondary incubation was then performed with goat a-mouse IgG Alexa Fluor 546 (1:400, Molecular Probes, A11030) for $1 \mathrm{~h}$ at RT. Along with the secondary incubation, DAPI (4,6-Diamidino-2-phenylindole, 1:1000, Sigma-Aldrich, D9542) was used to stain the nuclei of the cells. The samples were maintained in PBS without glucose until image acquisition, by automated fluorescence microscopy (Zeiss Axiovert 200M).

\subsection{Calcium detection by alizarin red staining}

The mineralization of the extracellular matrix, the last step of the osteogenic differentiation, was assessed at the $14^{\text {th }}$ and $21^{\text {st }}$ days of cell culture. The samples were fixed in 10\% formalin solution (Sigma-Aldrich, Germany) for 30 min, and then washed (5 $x 1 \mathrm{~min}$ ) in distilled water prior to incubation for $20 \mathrm{~min}$ in $2 \%$ Alizarin Red Solution (Merck, Germany). The Alizarin Red Solution was then washed in distilled water (5 x 10 min plus $1 \times$ overnight) and allowed to air-dry, before observation under optical microscopy (BX61, Olympus Corporation, Germany). 


\subsection{Image acquisition and quantification of biomarkers}

Images of the cells cultured on PCL surface-roughness gradients and controls were collected using the same microscopy settings to allow for the comparative expression of the osteogenic markers. Individual fluorescently labeled images were reconstructed into $10 \mathrm{~mm}$ gradient mosaics by the AxioVision 4.8.1 software. All images, including those collected by optical microscopy from Alizarin Red staining, were converted into the gray scale by ImageJ 1.46k software (Wayne Rasband, National Institutes of Health, USA) [52]. The histogram algorithm was subsequently applied to calculate the mean distribution of the gray values per $\mathrm{mm}^{2}$. The background of the collected images was also measured and subtracted from the raw data, so that the accurate intensity of the osteogenic expression could be assessed. In fluorescently labeled images, the background values were obtained from areas devoid of fluorochrome expression, while in Alizarin Red stained surface-roughness-gradient samples the background was measured at the flat PCL regions surrounding the hot-embossed gradient. The counting of cells was possible in Image $1.46 \mathrm{k}$ by the calculation of the average size of the nuclei, and subsequent application of a band-size filter (allowing for the exclusion of artifacts such as dust particles), in conjugation with the application of the watershed algorithm (which allowed for the separation of overlapping nuclei). The intensity of expression of ALP, COL1 and calcium staining was normalized to cell count, and the data were plotted in terms of mean \pm standard error. The standard error was calculated dividing the standard deviation of the mean by the square root of the population size. 


\subsection{Statistical Analysis}

A D'Agostino \& Pearson normality test was applied to determine the data normality. The results indicated that parametric tests should be used for the comparisons. A two-way ANOVA test followed by the Bonferroni posttest was used to analyze the effect of the substrate (TCPS or PCL surface-roughness) and culture time (in days) on the osteogenic markers ALP, COL1 and mineralization normalized to cell count. A one-way ANOVA followed by Dunnet's or Tukey's multiple comparison posttest was applied to compare the effect of different PCL roughness positions on the normalized expression values of the osteogenic markers at a given time point. The threshold significance level was set at 0.05 . Thus, ${ }^{*} p$ (probability) values inferior to 0.05 were considered statistically significant.

\section{Results}

\subsection{Characterization of the Roughness Gradient On the Polycaprolactone Surface} The PCL average roughness $\left(R_{a}\right)$, characterized by optical profilometry, progressively increased from the $1^{\text {st }}$ to the $10^{\text {th }} \mathrm{mm}$, from submicrometer to micrometer values $(\sim 0.5$ 4.7 $\mu \mathrm{m})$, while the mean spacing between the profile elements $\left(R S_{m}\right)$ steadily decreased from $\sim 214 \mu \mathrm{m}$ to $33 \mu \mathrm{m}$ (Figure 1), as previously analyzed [33].

\subsection{The Osteogenic Differentiation of hBM-MSCs On the PCL Roughness- Gradient In Dexamethasone-Deprived Medium}

The first signal of osteogenic commitment by the hBM-MSC population cultured on roughness gradients in dexamethasone-deprived (Dex-deprived) osteogenic induction (OI) medium was perceived at the $4^{\text {th }}$ day of culture. At this time point, the expression of 
alkaline phosphatase (ALP) on the roughness gradients was already evident, as assessed by immunodetection, and particularly notable at the micrometer roughnessaverage $(R a)$ side of the gradient (i.e., from the $5^{\text {th }}$ to the $10^{\text {th }} \mathrm{mm}$ ) (Figure 2). The expression of this early marker of osteogenesis gradually extended to the submicrometer end of the gradient until the last time point on day 21 (Figure 2 and 2.1A). The quantitative analysis of the ALP expression, normalized to cell count, confirmed that the earlier expression was obtained at day 4 on the micrometer side of the roughness gradient (especially at positions $5-9 \mathrm{~mm}, p>0.05$ ), followed by day 10 on both controls, and day 21 on the submicrometer side of the gradient (especially positions $1-3 \mathrm{~mm}$ ) (Figure 2.1B). Immunodetected signals of collagen type 1 (COL1) synthesis were subtle at early time points of the osteogenic commitment program, both on the gradient in Dex-deprived OIM and on the TCP+/- controls (Figure 2). Increasing intensity of the protein expression generally correlated with its morphological organization, which developed from globular, surrounding the cell nucleus, to fibrillar-like in the extracellular space (Figure 2 and 2.2A). Quantitatively, position $6 \mathrm{~mm}$ in the gradient showed consistently higher normalized COL1 expression than TCP+ (Figure 2.2.B). The TCP+ was also outperformed by the TCP- ( $p<0.001$, days 14 and 21$)$. Indeed, only positions 5 and 6 $\mathrm{mm}$ at days 14 and 21 showed a statistically similar $(p>0.05)$ or superior $(p<0.001)$ effect than TCP-. Already at day 14, the mineralization of the ECM, assessed by the staining of the samples with alizarin red, was perceptibly more intense at the micrometer end of the gradient, in comparison to the submicrometer end (Figure 4A). At day 21, the magnitude of the mineralization was greatest at positions 5 to $7 \mathrm{~mm}$ (Figure 
4B). In comparison, at the $21^{\text {st }}$ day, both controls $(\mathrm{TCP}+/-)$ showed statistically inferior performance than positions 5 to $10 \mathrm{~mm}$ in the gradient, but similar or higher performance than positions 1 to $4 \mathrm{~mm}$, at day 21 .

\subsection{The Osteogenic Differentiation of hBM-MSCs On the PCL Roughness- Gradient In Basal Growth Medium}

The hBM-MSC population cultured on tissue culture polystyrene in basal growth medium (TCP-B), i.e. lacking osteogenic-inducing factors, showed minor signals of ALP activity and negligible signals of intracellular COL1 expression (Figure 3, 3.1A, 3.2A). Also, no evidence of mineralization along the 21 days of culture was found (Figure 4A). In comparison, the surface-roughness gradient in basal growth medium showed differentiated activity levels of ALP activity, COL1 expression (both intracellular at early time points, and fibrillar in the extracellular space later on during the osteogenic process) and mineralization (Figures 3-4). The peak ALP activity occurred earlier on the surface-roughness gradient than on the positive control TCP+: $7^{\text {th }}$ versus $10^{\text {th }}$ day (Figure 3.1B). Moreover, the intensity of the ALP activity was $~ 55 \%$ stronger at positions 5 to $7 \mathrm{~mm}$ in the gradient than that obtained on TCP+ $(p<0.001)$.

On the surface-roughness gradient, the normalized COL1 synthesis and deposition globally increased from the second to the last time point (i.e., from the $4^{\text {th }}$ to the $21^{\text {st }}$ day) (Figure 3 and 3.2A). Position $5 \mathrm{~mm}$ showed higher effectiveness at supporting the COL1 expression than all other positions in the surface-roughness gradient and TCP+ $(p<0.001$, as seen in Figure 3B). Additionally, concomitantly with the fibrillar-like morphology of the COL1 fibers in the ECM, the globular arrangement of the protein surrounding the nucleus, seen at early time points, was again more distinguishable in 
the $21^{\text {st }}$ day (Figure $\left.3.2 \mathrm{~A}\right)$. This morphological evidence was considerably more pronounced in the surface-roughness gradient in basal growth medium than in the $\mathrm{TCP}+$.

The mineralization obtained in the surface-roughness gradient was higher at the micrometer end of the gradient $(6-10 \mathrm{~mm})$, from day 14 onwards (Figure $4 \mathrm{~A})$. The calcium deposition significantly increased from the 14 th to the 21 st day of culture $(p<$ $0.001)$ at the same positions and also significantly increased at position $5(p<0.05)$ (Figure 4B). Indeed, the mineralization observed at positions 5 to $7 \mathrm{~mm}$ was not significantly different at day $21(p>0.05)$. At the same time point, the TCP+ performance was superior to that observed at positions 1 to $3 \mathrm{~mm}$ in the gradient $(p<$ $0.05)$, and equivalent to the position $4 \mathrm{~mm}(p>0.05)$.

\subsection{Comparison of the Osteogenic Differentiation Intensity Occurring On Surface- Roughness Gradients In Dex-Deprived Medium and Basal Growth Medium} The peak ALP activity of the cell population on the surface-roughness gradients occurred earlier in Dex-deprived OIM than in basal growth medium (day 4 vs day 7), as seen in Figure 2 and 3. Although at different time points, the greatest level of ALP expression occurred in coincident positions in the surface-roughness gradients cultured in different cell-culture media - i.e., positions 5 to $7 \mathrm{~mm}$ (Figure 2.1B and 3.1B). Indeed, the ALP levels at these positions (day 4 in dex-deprived OI medium vs. day 7 in basal growth medium) was not significantly different $(p>0.05)$. Micrometer and submicrometer sides of the gradients also exposed significant differences in the morphology of the ALP expression, especially for the basal growth medium. In fact, in this cell culture medium, the ALP assumed a patchy-like morphology on the 
submicrometer positions (i.e., $1-4 \mathrm{~mm}$ ) until the last time point, while in Dex-deprived medium that morphology evolved to a cuboidal shape (Figure 2.1A and 3.1A).

The normalized COL1 expression by osteogenic-committed cells generally increased over time in both cell-culture media at all positions on the surface-roughness gradients (Figure 2-3). However, the COL1 expression was overall greatest at positions 5 and 6 $\mathrm{mm}$ in the gradients in basal growth medium and OIM, respectively. Nevertheless, at the last time point (day 21), positions 6 and $7 \mathrm{~mm}$, and 5 and $7 \mathrm{~mm}$ closely followed the most effective positions in basal growth medium and OIM medium, respectively (Figure 2.2B and 3.2B). From day 14 onwards, the mineralization was higher on the micrometer side for both gradient conditions, (Figure 4A). At the $21^{\text {st }}$ day, the weak performance at supporting the last stage of the osteogenic differentiation process was maintained for the submicrometer side of the gradients-especially at positions 1 and $2 \mathrm{~mm}$ in Dexdeprived OIM, and at positions 1 to $3 \mathrm{~mm}$ in basal growth medium. In contrast, the greatest effect was observed at position $6 \mathrm{~mm}$ in Dex-deprived OI medium, and position $5 \mathrm{~mm}$ in basal growth medium. However, in both cases, there were no significant differences between the effectiveness of positions 5 to $7 \mathrm{~mm}$ in the gradients $(p>0.05)$ (Figure 4B). However, there was greater mineralization in basal growth medium in comparison to the corresponding region in Dex-deprived OI medium $(p<0.001)$.

\section{Discussion}

It has been well established in the literature that the differentiation of human MSCs cultured in tissue culture polystyrene, used as a convenient reference material in vitro, can be influenced by different soluble factors in the cell-culture medium [25]. Over the last decades, successful synergies between (implantable) materials of interest and 
osteogenic-differentiation supplements in the culture medium have been thoroughly explored in the context of tissue engineering. However, those soluble supplements are often synthetic, not clearly replicating the signals that the cells sense in vivo, and their effect on the MSCs behavior varies alarmingly as a function of their concentration and interactions in the medium $[27,28,34]$. Thus, developing biomaterials for the purpose of bone-tissue regeneration, which are capable of guiding osteogenic differentiation per se would be extremely valuable [35]. The development of technology enabling a fine control of the surface topography of the materials has supported the growing interest in surface (sub-)microtopography as a modulator of the cell behavior $[33,36]$. However, the potential of surface topography as an alternative to soluble differentiation-inducers of osteogenesis has not been sufficiently explored. Gradients are a valuable tool, and enable the screening of a wide range of surface-roughness values that may facilitate or interfere with the intended fate of human MSCs [37]. The polymeric roughness gradient studied here avoided residual particles that are characteristic of many surfaceroughness fabrication techniques [38], by applying a hot-embossing replication technique previously described by our group [33]. Moreover, the variation in cell-culture conditions was significantly minimized by investigating different surface-average roughness in a gradient regime, instead of using discrete individual samples. With this approach, we validated the hypothesis that specific surface- $R_{a} / R S_{m}$ have the ability to trigger the osteogenic differentiation of hBM-MSCs on PCL, in incomplete osteogenic induction medium (OIM). Furthermore, even in the total absence of osteogenesisinducing supplements in the medium, such as dexamethasone, $\beta$-glycerophosphate, 
1,25-dihydroxyvitamine $D_{3}$ and ascorbate-2-phosphate, we observed the osteogenic commitment and differentiation of the hBM-MSC population.

Generally, the side of surface-roughness gradients having micrometer-scale roughness showed higher effectiveness at supporting osteogenesis, than the sub-micrometer side (i.e., positions $1-4 \mathrm{~mm}$ ). The differences were perceived not only quantitatively, but also morphologically. Unsurprisingly, the disadvantage was even more evident in the total absence of osteogenesis-inducing supplements in the medium. At these submicrometer-roughness positions, the ALP distribution on the cell surface assumed an elongated phenotype throughout the study, contrasting with the cuboidal cell phenotype characteristic of osteoblasts [39] seen on the remaining gradient positions. Position $6 \mathrm{~mm}\left(\mathrm{R}_{\mathrm{a}} \sim 1.5 \mu \mathrm{m} / \mathrm{RS}_{\mathrm{m}} \sim 79 \mu \mathrm{m}\right)$ in Dex-deprived OIM, and position $5 \mathrm{~mm}\left(\mathrm{R}_{\mathrm{a}}\right.$ $\sim 0.9 \mu \mathrm{m} / \mathrm{RS}_{\mathrm{m}} \sim 135 \mu \mathrm{m}$ ) in basal growth medium, consistently showed superior effectiveness at supporting the expression of the osteogenic markers ALP, COL1 and mineralization, in comparison to the tissue-culture polystyrene (TCP) controls $(\mathrm{TCP}+/-)$. The earlier ALP expression in the gradients cultured in Dex-deprived OIM, in comparison to the basal growth medium, suggested that the osteogenic cocktail plays the sole positive role on the acceleration of the induction of the cell population towards the osteogenic pathway. However, this did not translate into higher ALP activity in the Dex-deprived OIM. Indeed, the statistically insignificant difference between the peak expression levels of ALP on the gradients cultured in both culture media, suggests that the influence of specific surface roughness on the ALP activity can be greater than that of the culture media. This outcome may result from the absence of Dex from the incomplete OIM formulation, as the effectiveness of Dex is correlated with augmented 
ALP expression [40]. This correlation was, in fact, confirmed by the superior ALP activity obtained in TCP+ in comparison to that in TCP-. However, despite the presence of Dex in $\mathrm{TCP}+$, the peak activity of ALP on the micrometer side of the surface-roughness gradients cultured in Dex-deprived OIM and basal growth medium was comparatively stronger and earlier. Altogether, the data seems to confirm the significant ability of the surface roughness to support the osteogenic commitment of hBM-MSCs. Consistently, at specific surface-roughness positions (i.e., position 5 and $6 \mathrm{~mm}$ in basal growth medium and in Dex-deprived OIM, respectively) the COL1 expression continuously increased throughout the study, which is crucial for successful osteogenesis [41]. However, with the exception of COL1 synthesis, for which those positions significantly outperformed their gradient counterparts, positions 5 to $7 \mathrm{~mm}$ generally presented statistically insignificant differences, in both culture media, at supporting stronger ALP activity and degree of mineralization. Together, the results indicate a positive correlation between (early) robust ALP activity and (later) high mineralization, which is in good agreement with previous reports [42]. However, despite the fact that the ALP activity in both media is similar, the mineralization degree is significantly higher in basal growth medium. Thus, ALP and mineralization levels are not directly proportional. Interestingly, the presence of $\beta$-glycerophosphate and ascorbate-2-phosphate, sources of inorganic phosphates when hydrolyzed by ALP [43] did not translate into a higher degree of mineralization when cultured in Dex-deprived OIM. Indeed, the opposite occurred: positions 5 to $7 \mathrm{~mm}$ in the gradient cultured in basal growth medium appeared to support mineralization more efficiently. This observation is even more relevant since in TCP-B (i.e., TCP in basal growth medium) the contrary was observed, in agreement 
with the literature [44]. Together the data suggests that suggests that the hBM-MSC population can not only be directed towards the osteogenic pathway by physical environmental cues, but also that those features can robustly support the differentiation process even in the absence of strong soluble osteogenic inducers.

There are a limited number of reports in the literature studying the potential of surface topography to induce osteogenic commitment of hMSCs in the partial or total absence of soluble osteogenic inducers. Among them, nanotopographical pits in polycarbonate [45], nanoscale disordered cues in polymethylmethacrylate [31], and COL1-modified microrough titanium [46] were shown to positively influence the expression of osteogenic markers by hMSCs. However, the direct comparison of reports using different topographical features and substrates can be difficult, particularly if a standard control, such as TCP, is absent. We previously reported the effect of surface-roughness PCL gradients, identical to those studied in this work, in the osteogenic differentiation of hBM-MSCs cultured in complete OIM. In that study, positions 7 to $8 \mathrm{~mm}$ appeared to be the most effective [33]. The ALP activity, the COL1 expression and the mineralization on the gradient were, respectively, $\sim 3, \sim 1.3$ and $\sim 2$ times higher than the corresponding values on TCP+. By comparison, in Dex-deprived OIM and basal growth medium, the ALP activity was not as high as in the gradient in complete OIM, but it was still higher than the ALP activity in the TCP+. The results indicate the positive effect of dexamethasone in the ALP activity [47], as mentioned above, but also the positive synergy between that glucocorticoid and the topographical features in promoting ALP enhancement. In contrast, based on the direct comparison against the $\mathrm{TCP}+$, the levels of COL1 in the surface-roughness gradient cultured in complete OIM were inferior to 
those in Dex-deprived OI medium. The results seem to indicate that the effect of the surface-roughness is more intense at promoting the COL1 synthesis than the detrimental effect of Dex on the COL1, which is usually associated with higher concentrations of Dex [48,49]. Finally, the mineralization at the most effective positions of the gradients cultured in basal growth medium $(5$ to $7 \mathrm{~mm}$ ) showed as much of a superiority over the TCP+ as the best positions in the gradients cultured in complete OIM (position 7 and $8 \mathrm{~mm}$ ) [33]. The results occurred at a common position in the gradient (position $7 \mathrm{~mm}$ ) and indicate that specific surface roughness features are capable of inducing comparably strong osteogenic differentiation of hBM-MSCs, even in the absence of soluble osteogenesis-inducing agents and the corresponding synergistic interactions between surface and soluble factors. The influence of those specific surface

roughness features in the osteogenic differentiation may result from topography-induced modulation of the cell mechanotransduction, known to influence MSC differentiation [50], as those features may resemble the pits left by osteoclastic bone resorption, which ultimately induce bone deposition.

\section{Conclusions}

The use of PCL gradient platforms of continuously varying surface-roughness $\left(R_{a}\right.$ and $\left.R S_{m}\right)$ successfully allowed the determination of the most effective surface roughness, within the range explored here, for the osteogenic differentiation of hBM-MSCs cultured in different cell culture media. The effectiveness of the optimal surface-roughness range $\left(\mathrm{R}_{\mathrm{a}} \sim 0.9-2.1 \mu \mathrm{m} / \mathrm{RS}_{\mathrm{m}} \sim 135-71 \mu \mathrm{m}\right)$ cultured in dexamethasone-deprived osteogenic induction medium and in basal growth medium was significantly superior to that of the TCP control cultured in complete osteogenic induction medium (i.e., including 
dexamethasone in the formulation). The results revealed that specific surface- $R_{a}$ and $\mathrm{RS}$ m engineering can, per se, direct stem-cell fate on a clinically significant biodegradable polymer. The long-term stability of the surface-roughness as an osteogenic-inducing cue of stem cells, comparatively to the short-term availability of the osteogenic soluble supplements, which need to be replenished every 2 to 3 days, is of great interest. These findings present a valuable addition to the stem-cell differentiation toolbox, with potential applications in cell culture in vitro and in bone-tissue-engineered options involving clinically relevant materials with a similar molecular structure to that of PCL.

\section{Acknowledgements}

This work was supported by the Foundation for Science and Technology for the PhD grant of A.B. Faia-Torres (SFRH/BD/36476/2007). We are also grateful to the Swiss National Science foundation (SNSF) grant P300P3_154664 for providing funds to MC.

\section{Appendix A.}

Certain figures in this article, particularly Figures $2.1,2.2,3.1$ and 3.2 are difficult to interpret in black and white. The full color images can be found in the on-line version, at

\section{Figure legends:}

Figure 1. Graphing of the arithmetic mean deviation $\left(R_{a}\right)$ and of the mean spacing of the profile elements $\left(R_{S m}\right)$ within the $10 \mathrm{~mm}$ sampling length, following the ISO 4287 standard. The results are expressed in terms of the mean \pm standard error. 
Figure 2. Immunodetection of alkaline phosphatase (ALP), collagen type 1 (COL1) and nuclei on polycaprolactone (PCL) surface-roughness gradients cultured in dexamethasone (Dex)-deprived osteogenic induction medium (OIM), at days 4, 7, 14 and 21. Comparative expression of the original intensity of the fluorescence signal was analyzed against tissue culture polystyrene (TCP) in complete OIM (TCP+) and in dexdeprived OIM (TCP-). Scale bar represents $1 \mathrm{~mm}$ on the gradients and $0.5 \mathrm{~mm}$ on the remaining images.

Figure 2.1. A) Digital enhancement of the detected fluorescence signal of alkaline phosphatase (ALP) on zoomed in positions in the gradients and controls, in different media, at days 4, 7 and 21. OIM, osteogenic induction medium; Dex, dexamethasone; TCP, tissue culture polystyrene. B) Quantification of the ALP expression per $\mathrm{mm}^{2}$, normalized to the cell number in the same area, on PCL surface-roughness gradients cultured in Dex-deprived OIM over 21.

Figure 2.2. A) Digital enhancement of the detected fluorescence signal of collagen 1 (COL1) on zoomed in positions in the gradients and controls, in different media, at days 4, 14 and 21. OIM, osteogenic induction medium; Dex, dexamethasone; TCP, tissue culture polystyrene. B) Quantification of the COL1 expression per $\mathrm{mm}^{2}$, normalized to the cell number in the same area, on PCL surface-roughness gradients cultured in Dexdeprived OIM over 21 days. Quantitative comparison was performed against TCP in complete OIM (TCP+) and in dex-deprived OIM (TCP-). Statistical significance: $0.001<$ $p\left({ }^{*}\right)<0.05$. 
Figure 3. Immunodetection of alkaline phosphatase (ALP), collagen type 1 (COL1) and nuclei on polycaprolactone (PCL) surface-roughness gradients cultured in basal growth medium, at days $4,7,14$ and 21 . Comparative expression of the original intensity of the fluorescence signal was analyzed against tissue culture polystyrene (TCP) in complete OIM (TCP+) and in basal growth medium (TCP-). Scale bar represents $1 \mathrm{~mm}$ on the gradients and $0.5 \mathrm{~mm}$ on the remaining images.

Figure 3.1. A) Digital enhancement of the detected fluorescence signal of alkaline phosphatase (ALP) on zoomed in positions in the gradients and controls, in different media, at days 4, 7 and 21. OIM, osteogenic induction medium; TCP, tissue culture polystyrene. B) Quantification of the ALP expression per $\mathrm{mm}^{2}$, normalized to the cell number in the same area, on PCL surface-roughness gradients cultured in basal growth medium over 21 days. Quantitative comparison was performed against TCP in complete OIM (TCP+) and in basal growth medium (TCP-B). Statistical significance: $0.001<p\left(^{*}\right)$ $<0.05$.

Figure 3.2. A) Digital enhancement of the detected fluorescence signal of collagen 1 (COL1) on zoomed in positions in the gradients and controls, in different media, at days 4, 14 and 21. OIM, osteogenic induction medium; TCP, tissue culture polystyrene. B) Quantification of the COL1 expression per $\mathrm{mm}^{2}$, normalized to the cell number in the same area, on PCL surface-roughness gradients cultured in basal growth medium over 21 days. Quantitative comparison was performed against TCP in complete OIM (TCP+) and in dex-deprived OIM (TCP-). Statistical significance: $0.001<p\left({ }^{*}\right)<0.05$. 
Figure 4. A) Assessment of the mineralization pattern on polycaprolactone (PCL) surface-roughness gradients and on tissue culture polystyrene (TCP), at the $14^{\text {th }}$ and $21^{\text {st }}$ days of cell culture, using Alizarin Red staining. Scale bar represents $1 \mathrm{~mm}$ on the

gradients and $0.5 \mathrm{~mm}$ on the remaining images. B) Quantification of the final intensity of the alizarin red staining (i.e., after background intensity was removed from the analysis), normalized to the cell number per $\mathrm{mm}^{2}$, on the gradients cultured in dexamethasone (Dex)-deprived osteogenic induction medium (B, left) and in basal growth medium ( $B$, right) over 21 days. Quantitative comparison was performed against TCP in complete osteogenic induction medium (TCP+), in dex-deprived osteogenic induction medium (TCP-) and in basal growth medium (TCP-B). Statistical significance: $0.001<p\left(^{*}\right)<$ 0.05 .

\section{References}

[1] A. R. Amini, C. T. Laurencin, S. P. Nukavarapu, Bone tissue engineering: recent advances and challenges, Crit. Rev. Biomed. Eng. 40 (2012) 363-408.

[2] R. Langer, J. P. Vacanti, Tissue engineering, Science 260 (1993) 920-926.

[3] V. Stroganov, S. Zakharchenko, E. Sperling, A. K. Meyer, O. G. Schmidt, L. lonov, Biodegradable self-folding polymer films with controlled thermo-triggered folding, Adv. Funct. Mater. 24 (2014) 4357-4363.

[4] S. D. Kimmins, N. R. Cameron, Functional porous polymers by emulsion templating: recent advances, Adv. Funct. Mater. 21 (2011) 211-225.

[5] J. Lim, M. S. L. Chong, J. K. Y. Chan, S-H. Teoh, Polymer powder processing of cryomilled polycaprolactone for solvent-free generation of homogeneous bioactive tissue engineering scaffolds, Small 10 (2014) 2495-2502. 
[6] S. H. Oh, I. K. Park, J. M. Kim, J. H. Lee, In vitro and in vivo characteristics of pcl scaffolds with pore size gradient fabricated by a centrifugation method, Biomaterials 28 (2007) 1664-1671.

[7] Q. Zhang, Y. Jiang, Y. Zhang, Z. Ye, W. Tan, M. Lang, Effect of porosity on longterm degradation of poly (epsilon-caprolactone) scaffolds and their cellular response, Polym. Degrad. Stab 98 (2013) 209-218.

[8] B. D. Ulery, L. S. Nair, C. T. Laurencin, Biomedical applications of biodegradable polymers, J. Polym. Sci. Part B Polym. Phys. 49 (2011) 832-864.

[9] H. Petite, V. Viateau, W. Bensaïd, A. Meunier, C. de Pollak, M. Bourguignon, K. Oudina, L. Sedel, G. Guillemin, Tissue-engineered bone regeneration, Nat. Biotechnol. 18 (2000) 959-963.

[10] P. P. Carvalho, I. B. Leonor, B. J. Smith, I. R. Dias, R. L. Reis, J. M. Gimble, M. E. Gomes, Undifferentiated human adipose-derived stromal/stem cells loaded onto wetspun starch-polycaprolactone scaffolds enhance bone regeneration: nude mice calvarial defect in vivo study, J. Biomed. Mater. Res. A 102 (2014) 3102-3111.

[11] K. Rezwan, Q. Z. Chen, J. J. Blaker, A. R. Boccaccini, Biodegradable and bioactive porous polymer/inorganic composite scaffolds for bone tissue engineering, Biomaterials 27 (2006), 3413-3431.

[12] L. Bacakova, E. Filova, M. Parizek, T. Ruml, V. Svorcik, Modulation of cell adhesion, proliferation and differentiation on materials designed for body implants, Biotechnol. Adv. 29 (2011) 739-767. 
[13] A. Martins, E. D. Pinho, S. Faria, I. Pashkuleva, A. P. Marques, R. L. Reis, N. M. Neves, Surface modification of electrospun polycaprolactone nanofiber meshes by plasma treatment to enhance biological performance, Small 5 (2009) 1195-1206.

[14] D. F. Coutinho, M. E. Gomes, N. M. Neves, R. L. Reis, Development of micropatterned surfaces of poly(butylene succinate) by micromolding for guided tissue engineering, Acta Biomater. 8 (2011) 1490-1497.

[15] A. Martins, M. L. Alves da Silva, S. Faria, A. P. Marques, R. L. Reis, N. M. Neves, The influence of patterned nanofiber meshes on human mesenchymal stem cell osteogenesis, Macromol. Biosci.11 (2011) 978-987.

[16] C. N. Elias, Y. Oshida, J. H. C. Lima, C. A. Muller, Relationship between surface properties (roughness, wettability and morphology) of titanium and dental implant removal torque, J. Mech. Behav. Biomed. Mater. 1 (2008) 234-242.

[17] M. Bigerelle, K. Anselme, B. Noël, I. Ruderman, P. Hardouin, A. lost, Improvement in the morphology of ti-based surfaces: a new process to increase in vitro human osteoblast response, Biomaterials 23 (2002) 1563-1577.

[18] K. Anselme, M. Bigerelle, Statistical demonstration of the relative effect of surface chemistry and roughness on human osteoblast short-term adhesion, J. Mater. Sci. Mater. Med. 17 (2006) 471-479.

[19] N. J. Hallab, K. J. Bundy, K. O'Connor, R. L. Moses, J. J. Jacobs, Evaluation of metallic and polymeric biomaterial surface energy and surface roughness characteristics for directed cell adhesion, Tissue Eng. 7 (2001) 55-71.

[20] R. Gonzalez, C. B. Maki, J. Pacchiarotti, S. Csontos, J. K. Pham, N. Slepko, A. Patel, F. Silva, Pluripotent marker expression and differentiation of human second 
trimester mesenchymal stem cells, Biochem. Biophys. Res. Commun. 362 (2007) 491497.

[21] N. Stute, K. Holtz, M. Bubenheim, C. Lange, F. Blake, A. R. Zander, Autologous serum for isolation and expansion of human mesenchymal stem cells for clinical use Exp. Hematol. 32 (2004) 1212-1225.

[22] M. F. Pittenger, A. M. Mackay, S. C. Beck, R. K. Jaiswal, R. Douglas, J. D. Mosca, M. A. Moorman, D. W. Simonetti, S. Craig, D. R. Marshak, Multilineage potential of adult human mesenchymal stem cells, Science 284 (1999)143-147.

[23] N. Jaiswal, S. E. Haynesworth, A. I. Caplan, S. P. Bruder, Osteogenic differentiation of purified, culture-expanded human mesenchymal stem cells in vitro $\mathrm{J}$. Cell. Biochem. 64 (1997) 295-312.

[24] K. Anselme, A. Ponche, M. Bigerelle, Relative influence of surface topography and surface chemistry on cell response to bone implant materials. part 2: biological aspects, Proc. Inst. Mech. Eng. H 224 (2010) 1487-1507.

[25] C. Vater, P. Kasten, M. Stiehler, Culture media for the differentiation of mesenchymal stromal cells, Acta Biomater. 7 (2011) 463-477.

[26] A. Martins, A. R. C. Duarte, S. Faria, A. P. Marques, R. L. Reis, N. M. Neves, Osteogenic induction of hbmscs by electrospun scaffolds with dexamethasone release functionality, Biomaterials 31 (2010) 5875-5885.

[27] J. J. Alm, T. J. Heino, T. A. Hentunen, H. K. Väänänen, H. T. Aro, Transient 100 $\mathrm{nM}$ dexamethasone treatment reduces inter- and intraindividual variations in osteoblastic differentiation of bone marrow-derived human mesenchymal stem cells, Tissue Eng. Part C Methods 18 (2012), 658-666. 
[28] D. Hong, H.-X. Chen, Y. Xue, D.-M. Li, X.-C. Wan, R. Ge, J.-C. Li, J. osteoblastogenic effects of dexamethasone through upregulation of taz expression in rat mesenchymal stem cells, Steroid Biochem. Mol. Biol. 116 (2009) 86-92.

[29] A. Nair, J. Shen, P. Lotfi, C.-Y. Ko, C. C. Zhang, L. Tang, Biomaterial implants mediate autologous stem cell recruitment in mice, Acta Biomater.7 (2011) 3887-3895. [30] R. Tasso, F. Fais, D. Reverberi, F. Tortelli, R. Cancedda, The recruitment of two consecutive and different waves of host stem/progenitor cells during the development of tissue-engineered bone in a murine model, Biomaterials 31 (2010) 2121-2129.

[31] M. J. Dalby, N. Gadegaard, R. Tare, A. Andar, M. O. Riehle, P. Herzyk, C. D. W. Wilkinson, R. O. C. Oreffo, The control of human mesenchymal cell differentiation using nanoscale symmetry and disorder, Nat. Mater. 6 (2007) 997-1003.

[32] A. Wilkinson, R. N. Hewitt, L. E. McNamara, D. McCloy, R. M. D. Meek, M. J. Dalby, Biomimetic microtopography to enhance osteogenesis in vitro, Acta Biomater. 7 (2011) 2919-2925.

[33] A. B. Faia-Torres, S. Guimond-Lischer, M. Rottmar, M. Charnley, T. Goren, K. Maniura-Weber, N. D. Spencer, R. L. Reis, M. Textor, N. M. Neves, Differential regulation of osteogenic differentiation of stem cells on surface roughness gradients. Biomaterials 35 (2014) 9023-9032.

[34] J. Li, N. Zhang, X. Huang, J. Xu, J. C. Fernandes, K. Dai, X. Zhang, Dexamethasone shifts bone marrow stromal cells from osteoblasts to adipocytes by c/ebpalpha promoter methylation, Cell Death Dis. 4 (2013) 832-843.

[35] J. M. Anderson, J. B. Vines, J. L. Patterson, H. Chen, A. Javed, H.-W. Jun, Osteogenic differentiation of human mesenchymal stem cells synergistically enhanced 
by biomimetic peptide amphiphiles combined with conditioned media, Acta Biomater. 7 (2011) 675-682.

[36] A. Marino, G. Ciofani, C. Filippeschi, M. Pellegrino, M. Pellegrini, P. Orsini, M. Pasqualetti, V. Mattoli, B. Mazzolai, Two-photon polymerization of sub-micrometric patterned surfaces: investigation of cell-substrate interactions and improved differentiation of neuron-like cells, ACS Appl. Mater. Interfaces 5 (2013) 13012-13021. [37] P. Y. Wang, L. R. Clements, H. Thissen, A. Jane, W. Tsai, N. H. Voelcker, Screening Mesenchymal Stem Cell Attachment and Differentiation on Porous Silicon Gradients, Adv. Funct. Mater. 22 (2012) 3414-3423.

[38] T. Papadopoulos, A. Tsetsekou, G. Eliades, Effect of aluminium oxide sandblasting on cast commercially pure titanium surfaces, Eur. J. Prosthodont. Restor. Dent. 7 (1999) 15-21.

[39] J. Lee, A. A. Abdeen, K. A. Kilian, Rewiring mesenchymal stem cell lineage specification by switching the biophysical microenvironment, Sci. Rep. 4 (2014) 51885196.

[40] K. H. Wei, G. X. Pei, L. Zheng, The effects of dexamethasone on biological characteristics of bone marrow stromal cells. J. Reparative Reconstr. Surg. 15 (2001) 232-234.

[41] M. J. Glimcher, Metabolic Bone Disease and Clinically Related Disorders, Academic Press, London, 1998.

[42] E. E. Golub, K. Boesze-Battaglia, The role of alkaline phosphatase in mineralization, Curr. Opin. Orthop. 18 (2007) 444-448. 
[43] Y. L. Chang, C. M. Stanford, J. C. Keller, Calcium and phosphate supplementation promotes bone cell mineralization: implications for hydroxyapatite (ha)enhanced bone formation. J. Biomed. Mater. Res.52 (2000) 270-278.

[44] C. Hildebrandt, H. Büth, H. Thielecke, Influence of cell culture media conditions on the osteogenic differentiation of cord blood-derived mesenchymal stem cells, Ann. Anat. Anat. Anz. 191 (2009) 23-46.

[45] E. Kingham, K. White, N. Gadegaard, M. J. Dalby, R. O. C. Oreffo, Nanotopographical cues augment mesenchymal differentiation of human embryonic stem cells, Small 9 (2013) 2140-2151.

[46] M. Morra, C. Cassinelli, G. Cascardo, D. Bollati, R. R. Baena, Gene Expression of markers of osteogenic differentiation of human mesenchymal cells on collagen imodified microrough titanium surfaces, J. Biomed. Mater. Res. A 96 (2011) 449-455. [47] Y. Xiao, V. Peperzak, L. van Rijn, J. Borst, J. D. de Bruijn, Dexamethasone treatment during the expansion phase maintains stemness of bone marrow mesenchymal stem cells, J. Tissue Eng. Regen. Med. 4 (2010) 374-387. [48] S. Advani, D. LaFrancis, E. Bogdanovic, P. Taxel, L. G. Raisz, B. E. Kream, Dexamethasone Suppresses In Vivo Levels of Bone Collagen Synthesis in Neonatal Mice, Bone 20 (1997) 41-46.

[49] J. Li, X. Wang, C. Zhou, L. Liu, Y. Wu, D. Wang, H. Jiang, Glucocorticosteroid treatment delays early healing of a mandible wound by inhibiting osteogenic differentiation, Injury 43 (2012) 1284-1289. 
[50] B. K. Teo, S. T. Wong, C. K. Lim, T. Y. Kung, C. H. Yap, Y. Ramagopal, L. H.

Romer, E. K. Yim, Nanotopography modulates mechanotransduction of stem cells and induces differentiation through focal adhesion kinase, ACS Nano. 7 (2013) 4785-98.

[51] D. Studer, S. Lischer, W. Jochum, M. Ehrbar, M. Zenobi-Wong, K. Maniura-

Weber, Ribosomal protein I13a as a reference gene for human bone marrow-derived

mesenchymal stromal cells during expansion, adipo-, chondro-, and osteogenesis, Tissue Eng. Part C Methods 18 (2012) 761-771.

[52] C. A. Schneider, W. S. Rasband, K. W. Eliceiri, NIH Image to ImageJ: 25 Years of Image Analysis, Nat. Methods 9 (2012) 671-675. 


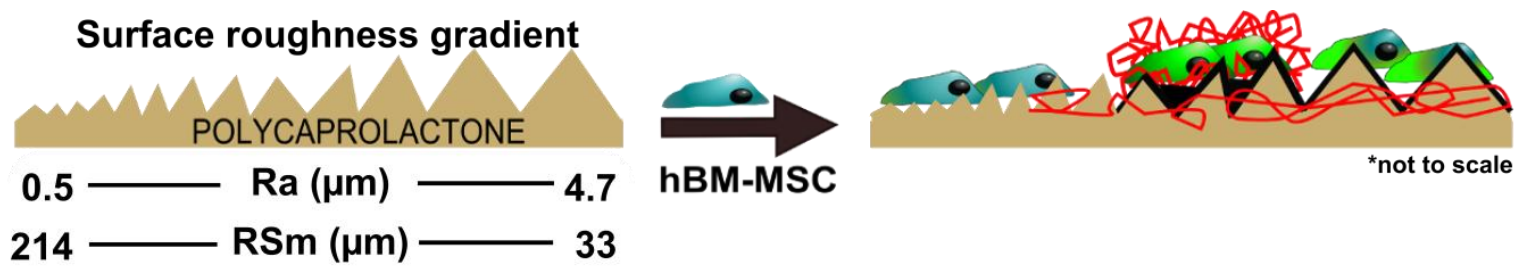

negligible ALP expression by the (osteo-commited) hBM-MSC population medium ALP expression by the (osteo-commited) hBM-MSC population

- strong ALP expression by the (osteo-commited) hBM-MSC population

enfellagen type 1 deposition

mineralization 
Figure 1:

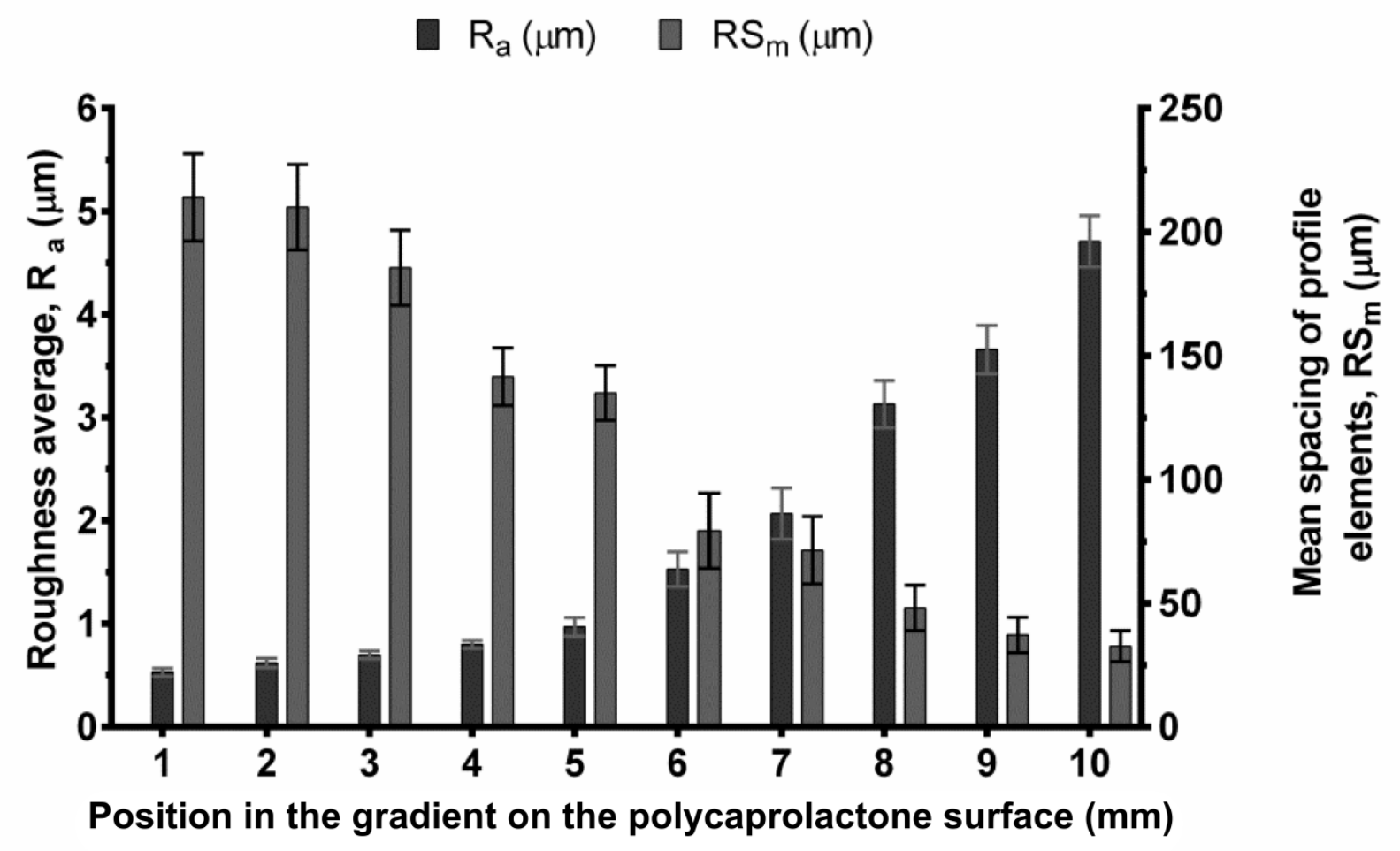


Figure 2:

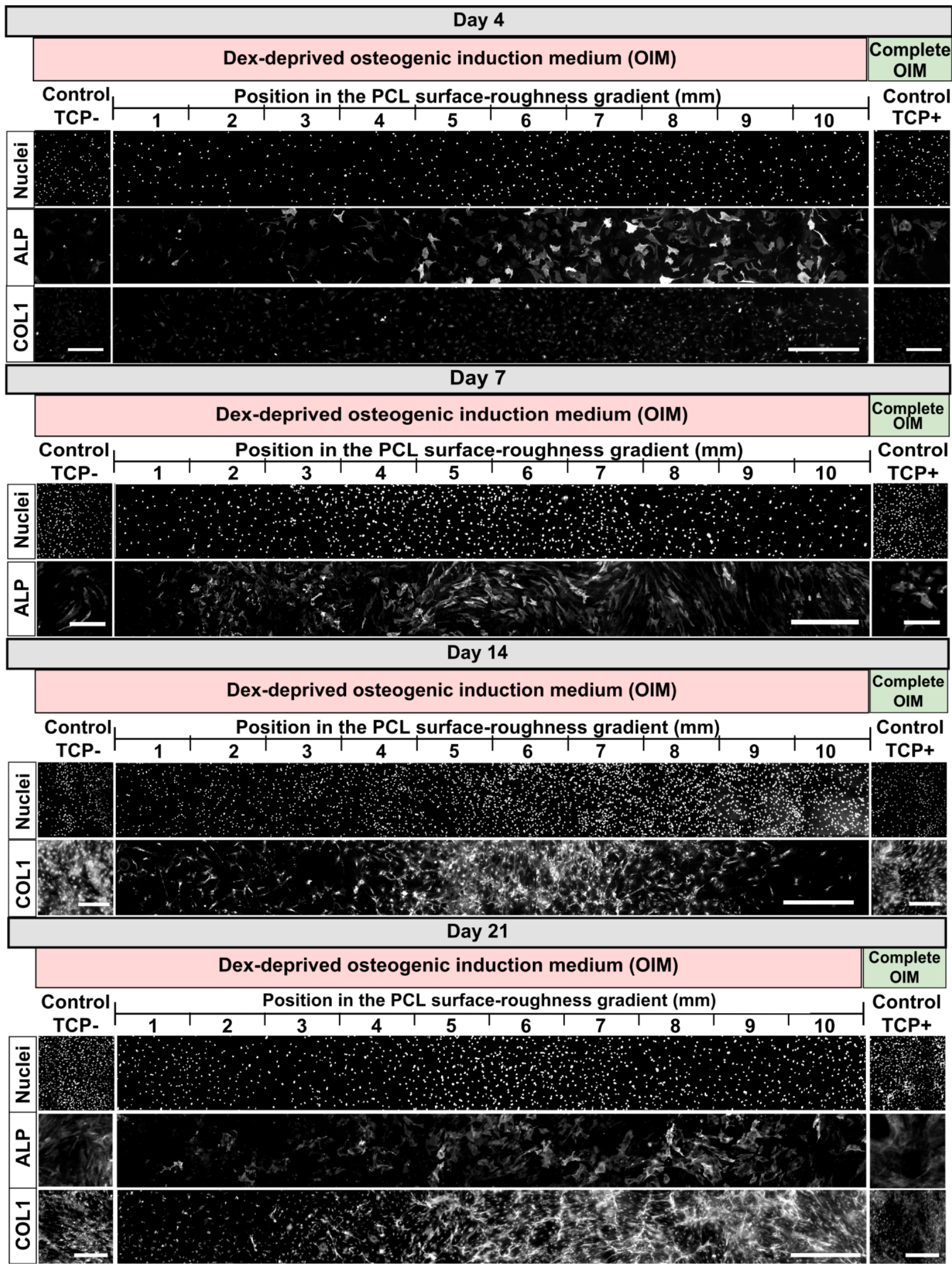


Figure 2.1:

A.

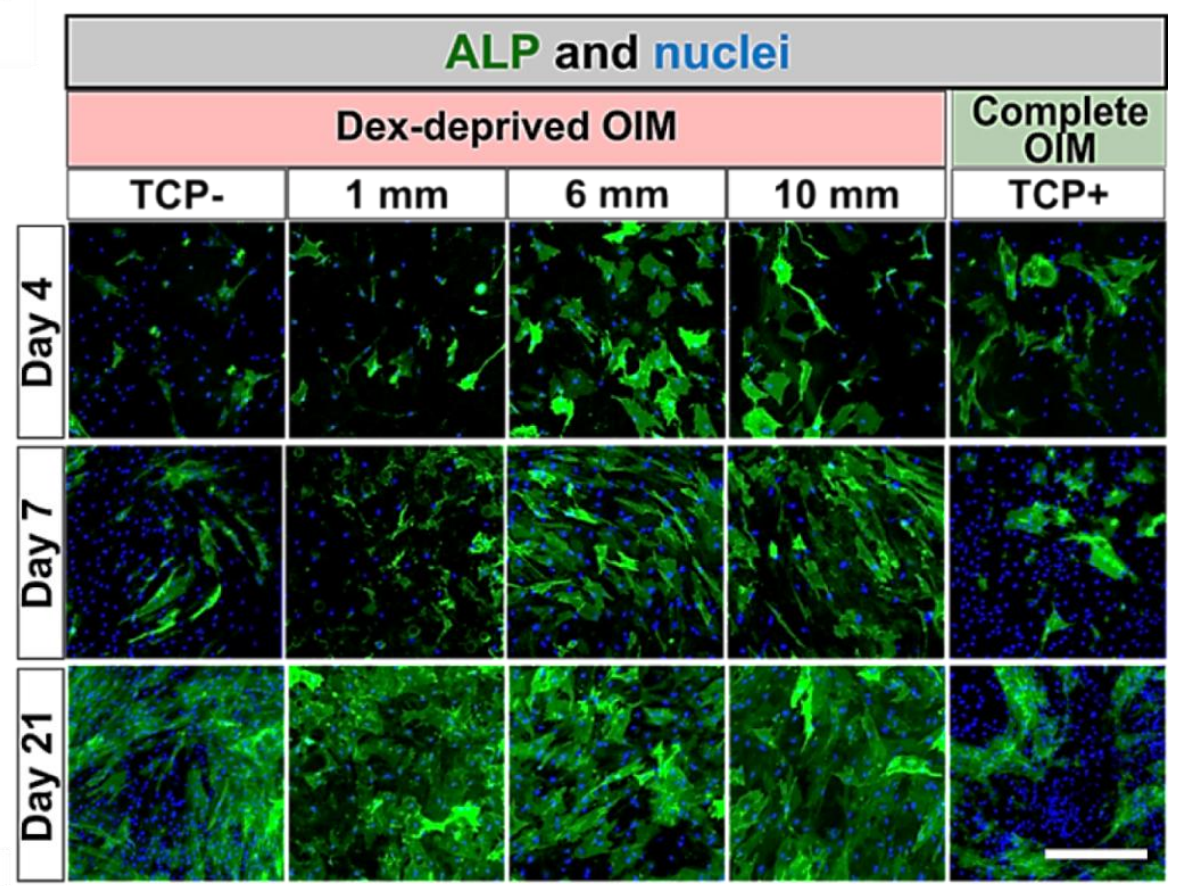

B.
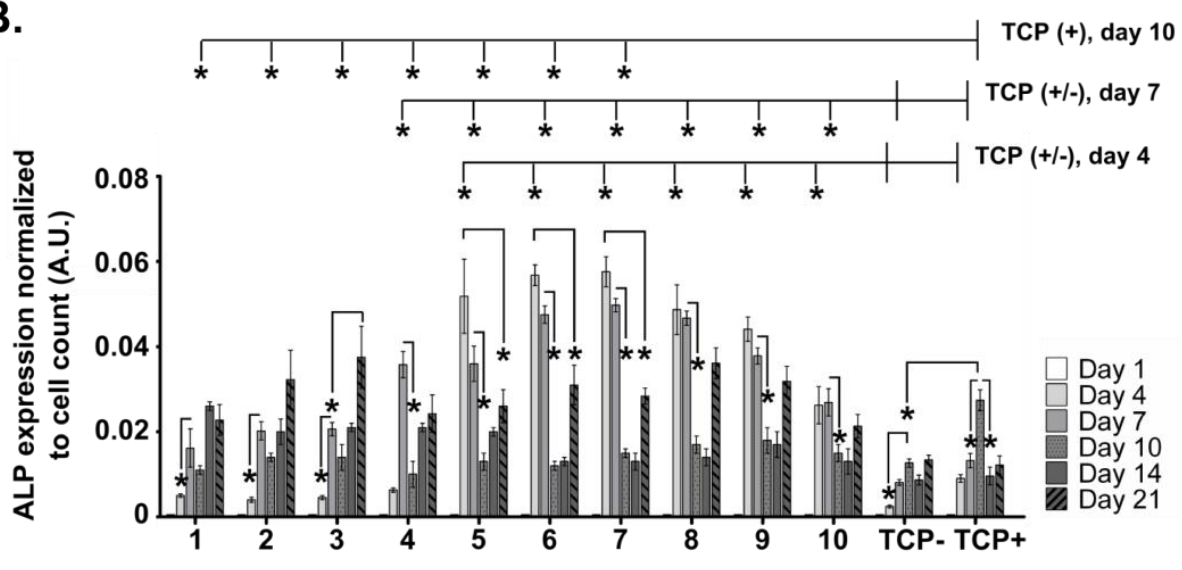

Position on the surface-roughness gradient and on TCP $(\mathrm{mm})$ 
Figure 2.2:

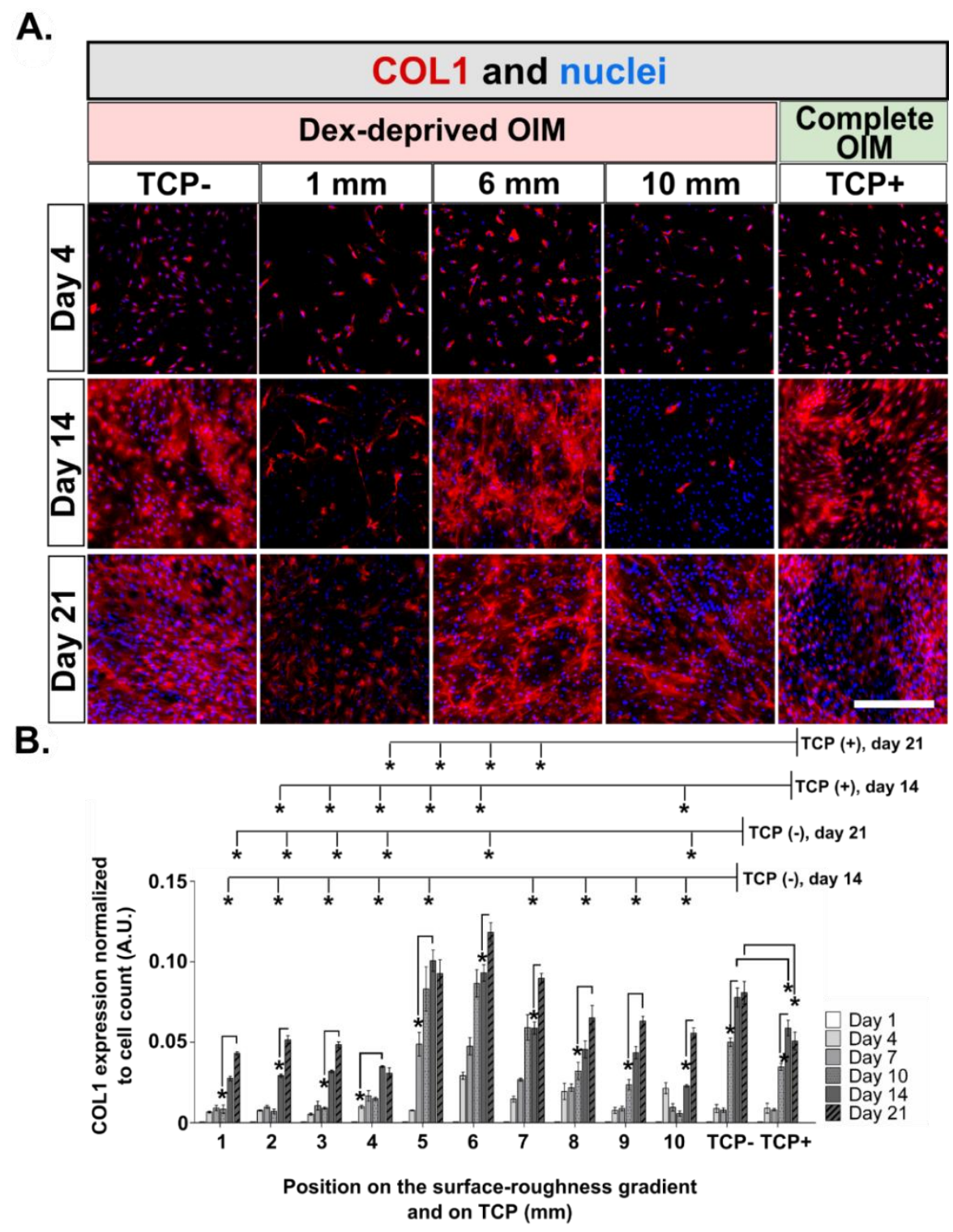


Figure 3:

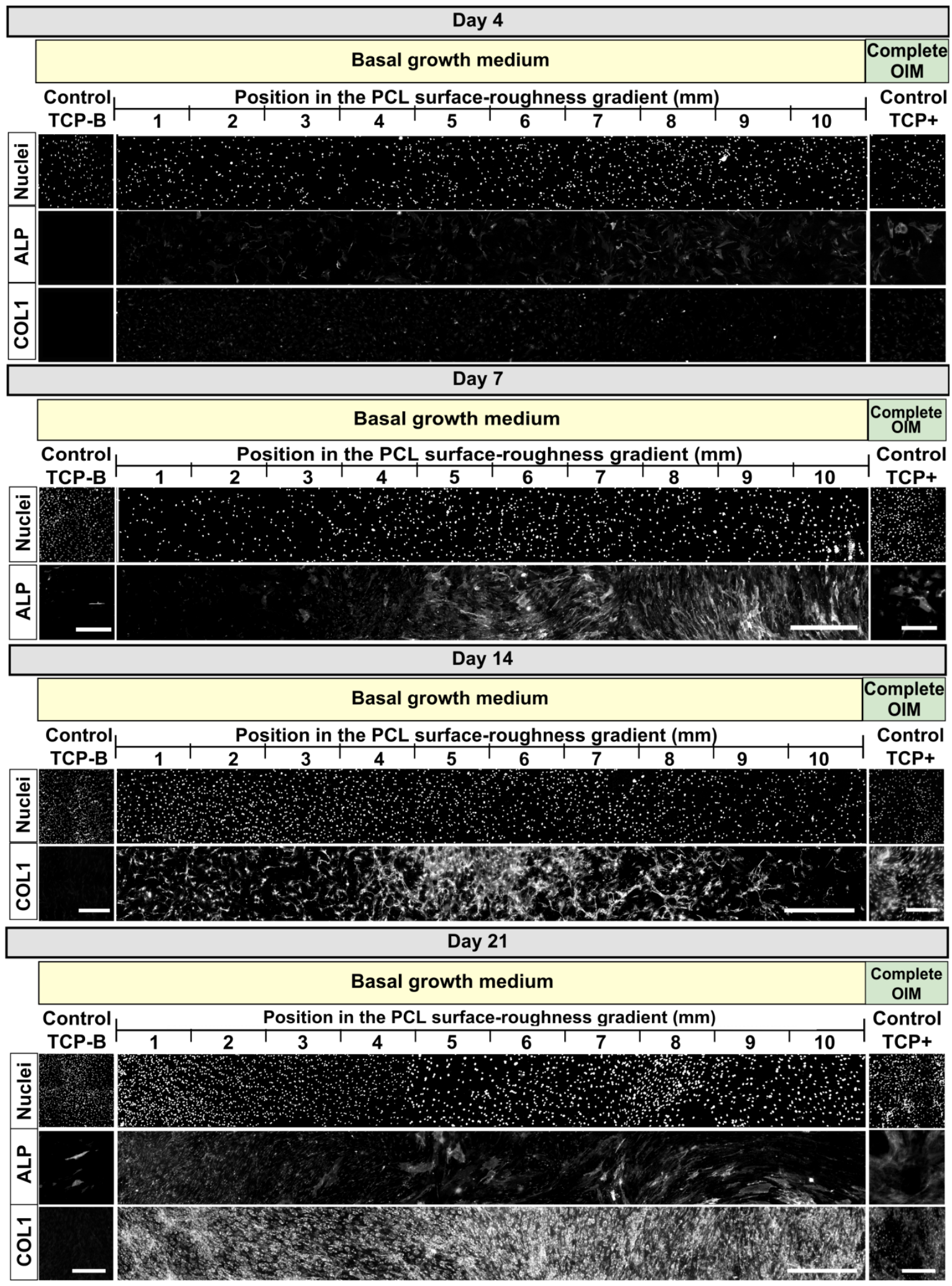


Figure 3.1:

A.

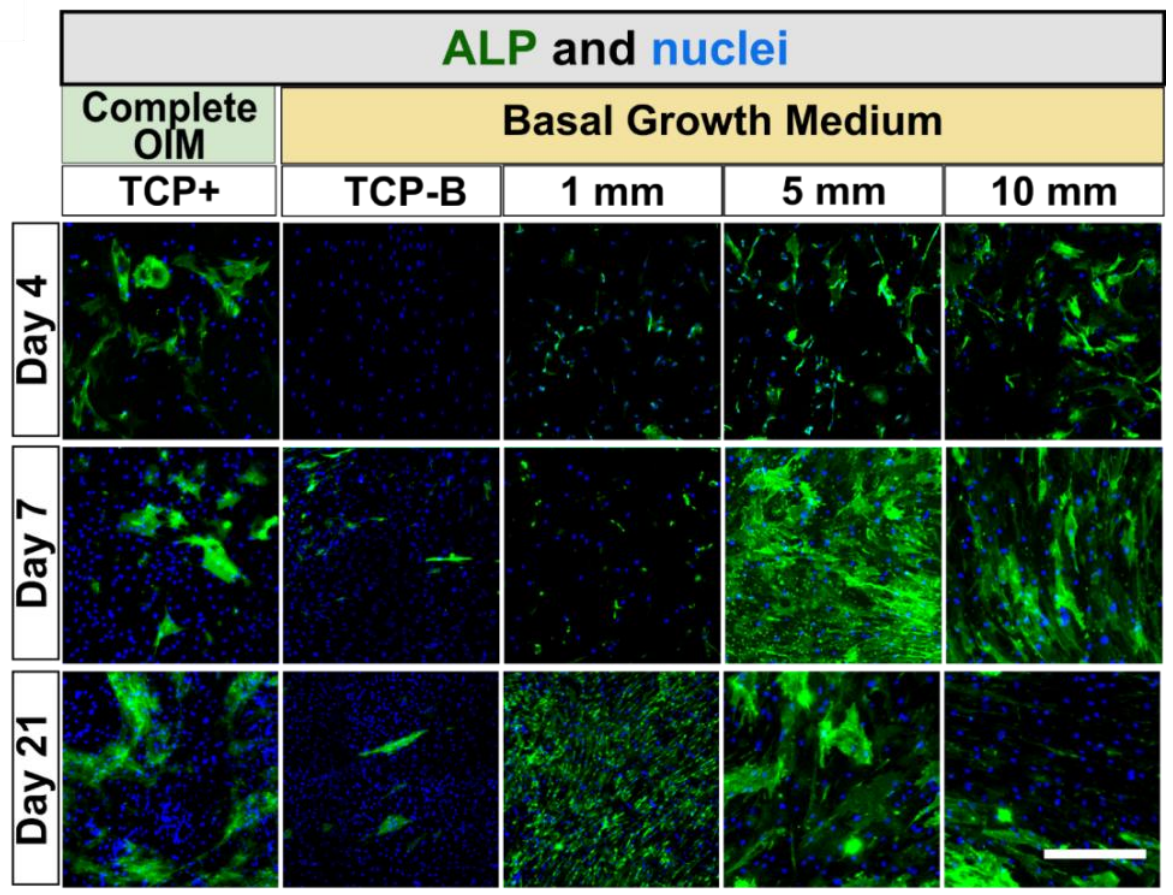

B.

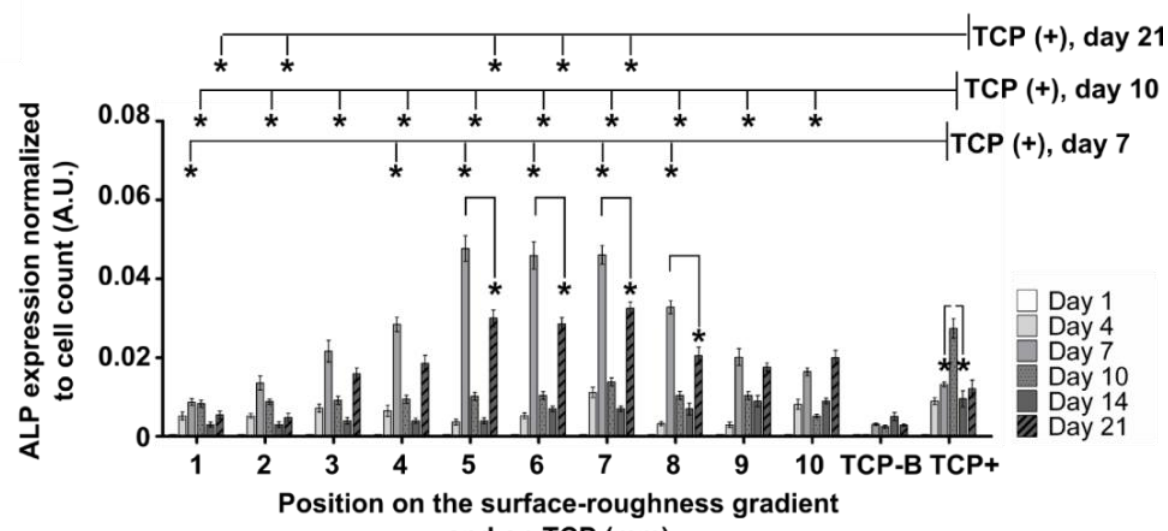
and on TCP $(\mathrm{mm})$ 
Figure 3.2:

A.

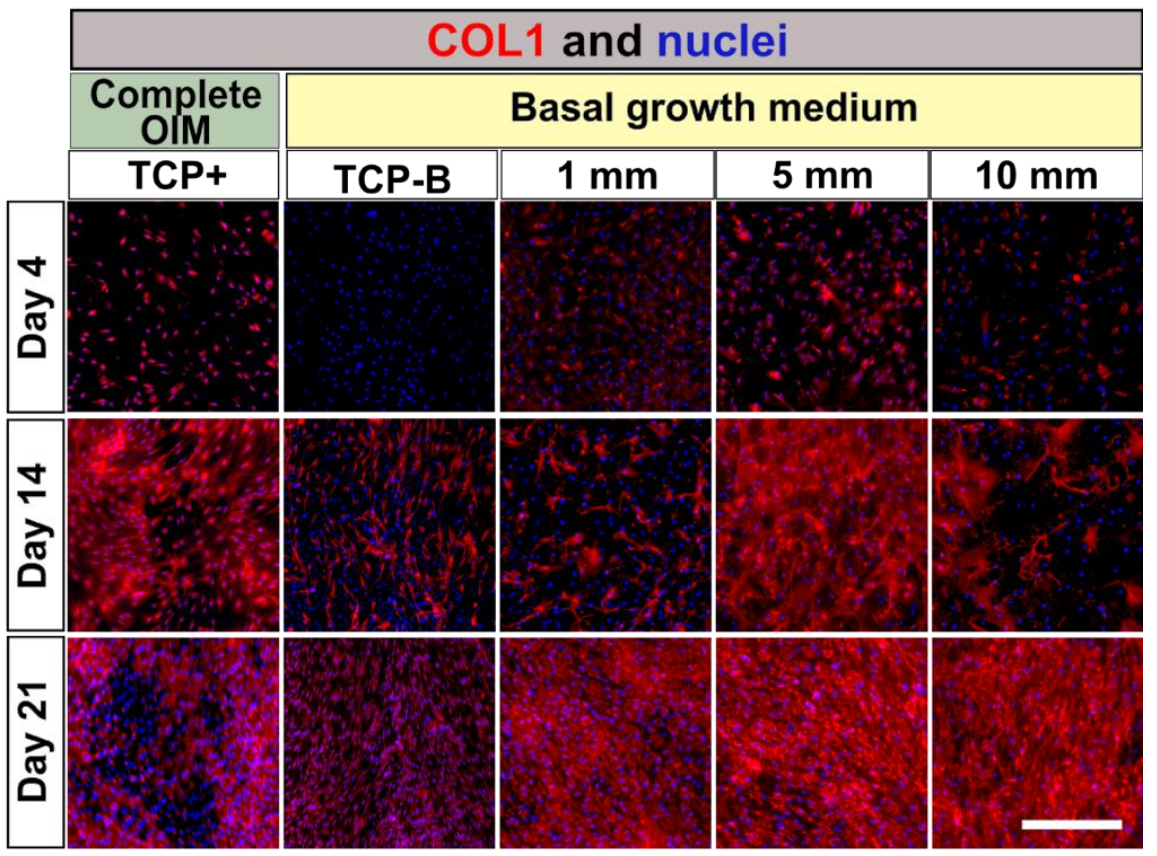

B.

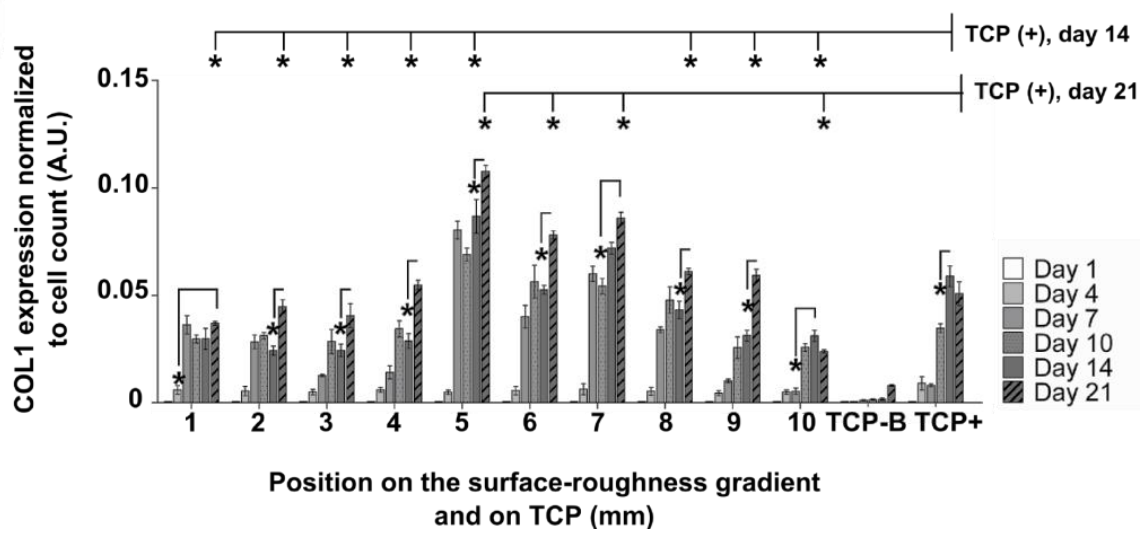




\section{Figure 4:}

A)

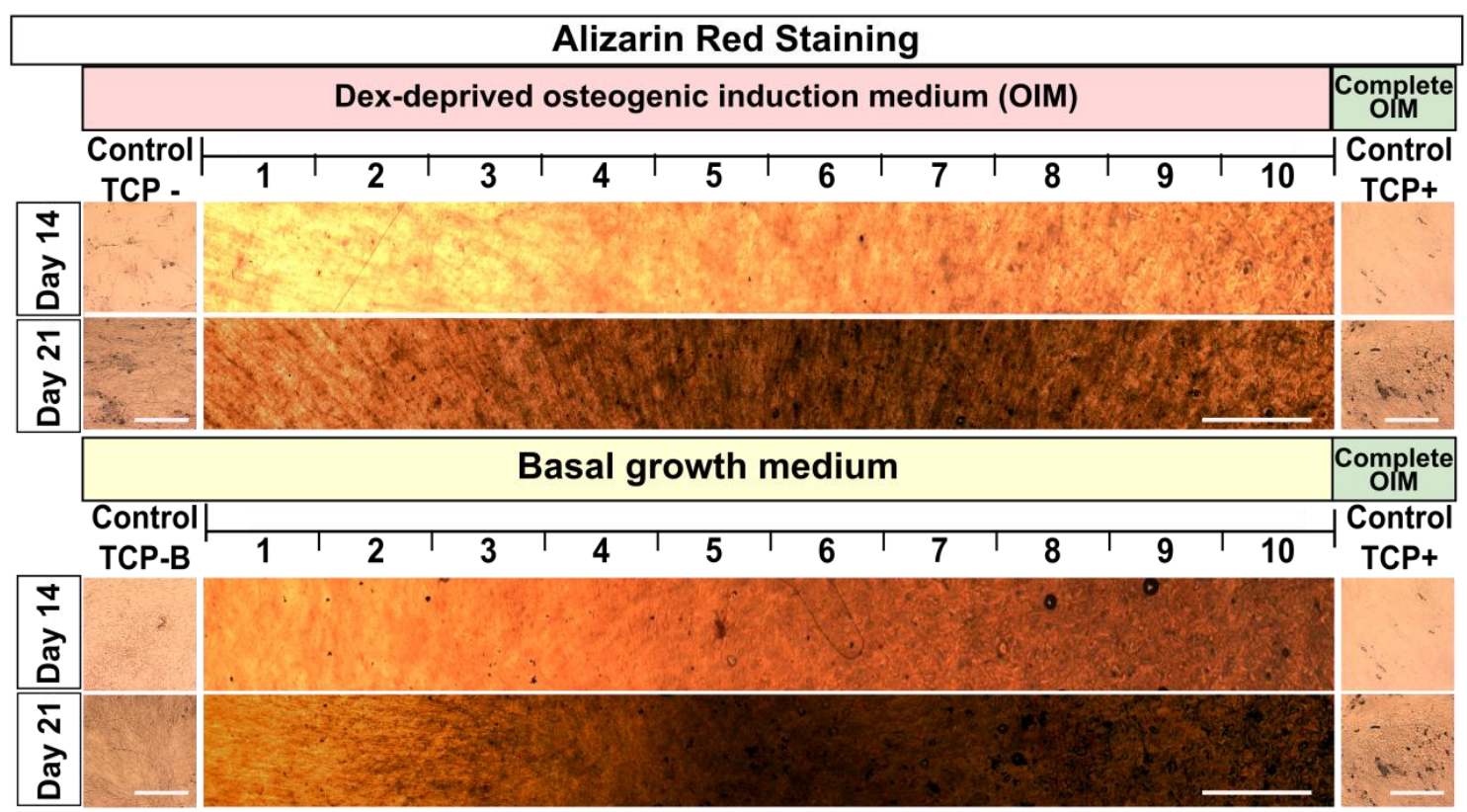

B)
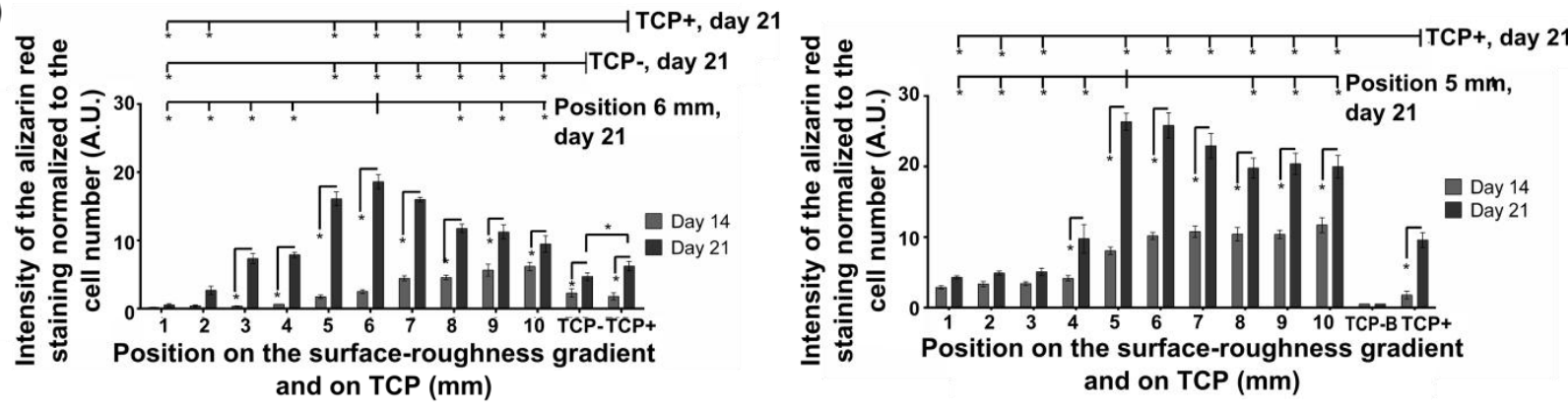